\title{
Impact of blue-depleted white light on pupil dynamics, melatonin suppression and subjective alertness following real-world light exposure
}

\author{
Jamie M. Zeitzer ${ }^{1,2^{*}}$ (D, Raymond P. Najjar ${ }^{1,3}$, Cheng-Ann Wang ${ }^{1}$ and Mirelle Kass ${ }^{1,4}$
}

\begin{abstract}
Background: The non-image forming system, which conveys light information to circadian and sleep centers in the brain, is optimized to respond to short wavelengths of light (blue). Exposure to white light with reduced blue content can cause lower than expected circadian and sleep responses. These findings, however, come from controlled laboratory conditions that may not be entirely accurate when attempting to apply them to most realworld settings. It was our intention to examine whether, under ecologically-valid circumstances, a blue-depleted white light had a diminished impact on sleep and circadian functions as compared to an equiluminant white light.

Methods: In Study 1, seven healthy, young individuals were exposed to a series of one-minute light pulses $(32,100$ or $140 \mathrm{~lx})$ produced either by a standard white light emitting diode (LED) or an LED light with reduced blue content. Pupil responses were measured with an infrared pupillometer. In Study 2, ten healthy, young individuals participated in two overnight evaluations. On one of the nights, participants received three hours of 150 Ix of a standard white LED starting at habitual bedtime. The protocol on the alternate night was identical except an LED with reduced blue content was used (both lights were identical to those used in Study 1). Saliva samples were collected every 20-30 min for determination of melatonin concentrations and subjective sleepiness was assessed hourly with the Stanford Sleepiness Scale. In both studies, pre-light exposure baseline was real-world ambulatory light exposure.
\end{abstract}

Results: Study 1. The post-illumination pupil response (PIPR) to $32 \mathrm{~lx}$ was increased in response to the standard as compared to blue-depleted LED ( $p<0.05$, paired t-test). PIPR did not differ between lighting conditions at higher illuminances. Study 2. Neither salivary melatonin concentrations nor subjective sleepiness scores were different between lighting conditions.

Conclusions: While the absence or reduction of blue light has the physiologic capacity to reduce the impact of light on non-image forming photoreceptive functions, under a pre-exposure lighting environment closer to that which is found in the real world, no such differences are observed except for pupil responses to moderately dim light.

Trial registration: ClinicalTrials.gov \#NCT02936674, NCT02636140.

Keywords: Light, Blue light, Short wavelength light, Melatonin, Alertness, Pupil

\footnotetext{
* Correspondence: jzeitzer@stanford.edu

'Department of Psychiatry and Behavioral Rhythms, Stanford University,

Stanford, CA 94305, USA

${ }^{2}$ Mental Illness Research Education and Clinical Center, VA Palo Alto Health

Care System, Palo Alto, CA 94304, USA

Full list of author information is available at the end of the article
} 


\section{Background}

While retinal photoreception is mostly thought of as the basis of "image formation", the retina underlies a number of non-image forming functions as well. Notable among these are synchronizing the timing of the circadian clock (Czeisler et al., 1989), suppressing pineal melatonin production (Zeitzer et al., 2000), increasing alertness (Cajochen et al., 2000), and changing pupil size (Alpern \& Campbell, 1962). In mammals, while rods and cones are the main contributors to conscious visual perception, a combination of rods, cones, and melanopsin contribute to non-image forming photoreception. Melanopsin is a light-absorbing pigment expressed in the intrinsically photosensitive retinal ganglion cells (ipRGCs) and conveys the eponymous intrinsic photosensitivity (Berson et al., 2002). ipRGCs project widely to the brain (notably the hypothalamus) to convey information about the overall intensity of light (Nelson \& Takahashi, 1991).

Melanopsin has a peak sensitivity in the blue range of the light spectrum (Newman et al., 2003) and the spectral sensitivity of sustained melatonin suppression also peaks in the blue light range $(\sim 460 \mathrm{~nm})$ (Brainard et al., 2001). The peak photopic sensitivity for conscious image formation is, however, in the green portion of the spectrum $(555 \mathrm{~nm})$. As such, it has been theorized that exposure to broad spectrum white light that has been depleted of or has minimal short wavelengths (blue light) would not alter conscious visual perception but would minimally activate non-image forming photoreceptive functions (McBean et al., 2016). This would be notable for extended (hours) light stimuli as the response to shorter light stimuli is likely more driven by cones (Gooley et al., 2010). In one study of spectrally-altered polychromatic light, blue-depleted white light ( $239 \mathrm{~lx}$, normal room lighting) was not different from normal white light in terms of its impact on sleep latency, melatonin suppression, and sleepiness (Santhi et al., 2011). In a separate study, authors found that $50 \mathrm{~lx}$ of blue-depleted white light ( $50 \mathrm{~lx}$, low room lighting) was less effective at melatonin suppression and caused less enhancement of electroencephalographic measures of alertness than $50 \mathrm{~lx}$ of normal white light (Rahman et al., 2017). In this latter study, however, participants spent $8 \mathrm{~h}$ in moderate room lighting $(\sim 88 \mathrm{~lx})$ prior to receiving the experimental light which likely sensitized the responses to the low intensity light (Smith et al., 2004; Chang et al., 2011).

The purpose of this experiment was to test under real-world conditions whether a commercially-available LED-based lamp that emitted a broad spectrum white light had greater impacts on pupil function, subjective alertness and melatonin suppression than a commerciallyavailable LED-based bulb that emitted a broad spectrum, blue-depleted white light.

\section{Methods}

\section{Study 1}

We examined seven participants (four male, 3 female) during a single 3 -h session. Five were Caucasian, one was Asian, and one identified as multiple races. They were aged 21-29 (26 \pm 3.4 years, mean \pm SD). Participants came to the laboratory between 4 and $7 \mathrm{~h}$ after their typical wake time, following a night during which they had at least $7 \mathrm{~h}$ in bed allotted towards sleep (selfreported). All participants were in good self-reported physical and mental health, not depressed $(<28$ on the Center for Epidemiologic Studies Depression Scale (Radloff, 1977)), without sleep disorders $(<6$ on the Pittsburgh Sleep Quality Index (Buysse et al., 1989)), of intermediate chronotype (Horne \& Östberg, 1976), were not regular smokers, and did not have an alcohol use disorder $(<20$ on the Alcohol Use Disorders Identification Test; lack of proximal alcohol use was confirmed upon entry to the laboratory with salivary alcohol test). All participants had normal color vision (Ishihara plate test (Ishihara, 2007)), lacked self-reported ocular pathologies, and did not use medications that impacted ocular function or pupil size. Female participants were not pregnant, as confirmed upon entry to the laboratory with a urinary pregnancy test. All procedures were approved by the Stanford University Institutional Review Board and conformed to the principals outlined in the Declaration of Helsinki.

Following completion of screening questionnaires and determination of eligibility, participants took part in an approximately three-hour examination of their pupil responses to light. During this examination, participants were seated and placed their chin on a chin rest and rested their forehead on a temple bar, all of which fixed the distance between the eyes and an experimental light source both within and between participants. A headmounted infrared eye tracker (ViewPoint USB-60 $\times 3$ Binocular Pupillometry system, Arrington Research, Scottsdale AZ) was placed on the participant's head and was used to record pupil size (recorded at $60 \mathrm{~Hz}$ ) throughout the study. Once the eye tracker was in place, participants were exposed to $30 \mathrm{~min}$ of darkness. Following this dark adaptation, participants were exposed to a series of 1-min light pulses, each of which were separated by $10 \mathrm{~min}$ of darkness (to allow for partial dark adaptation of rhodopsin), that were produced by one of two lamps - (1) a standard broad spectrum (white) LED (EcoSmart BR30, $2700 \mathrm{~K}$, color rendering index $=95$, Home Depot, Atlanta GA) the spectrum of which is generated by blue LED exciting phosphors, or (2) a bluedepleted white LED (BlueFree in a $9.525 \mathrm{~cm}$ diameter bulged reflector casing, $2700 \mathrm{~K}$, color rendering index = 78 , Soraa, Fremont CA) that has a significantly reduced short wavelength light (blue) component (Fig. 1) as its 


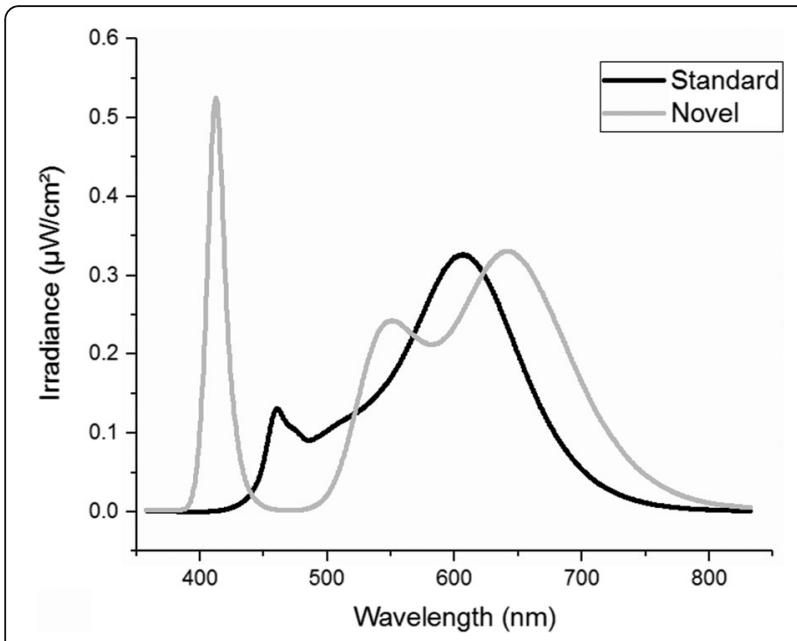

Fig. 1 Spectral output of the blue-depleted (grey) and standard (black) white LED lamps. Irradiance was measured with a research spectroradiometer (ILT-900R, International Light Technologies, Peabody MA)

spectrum is generated by violet LED exciting phosphors. Lamps were placed behind an ultravioletfiltering, clear plastic panel fitted with neutral density filters (Roscolux neutral gray, \#398; Rosco, Stamford $\mathrm{CT}$ ) and a diffuser (Roscolux tough white diffusion \#116; Rosco, Stamford CT). The two lamps were each calibrated to produce three different illuminances: $32 \mathrm{~lx}\left(\log\right.$ photon flux: $\left.13.6 \log _{10}\left(1 / \mathrm{cm}^{2} / \mathrm{s}\right)\right), 100 \mathrm{~lx}(\log$ photon flux: $\left.14.1 \log _{10}\left(1 / \mathrm{cm}^{2} / \mathrm{s}\right)\right)$, and $140 \mathrm{~lx}$ (log photon flux: $14.2 \log _{10}\left(1 / \mathrm{cm}^{2} / \mathrm{s}\right)$ ) (corneal illuminances confirmed in situ with an ILT1700 Research Photometer, International Light Technologies, Peabody MA; photon flux determined with ILT-900R, International Light Technologies, Peabody MA and converted using the Lucas toolbox (Lucas et al., 2014)). Light was presented in a diffuse circle at a $21^{\circ}$ visual angle. All illuminances used were in the photopic range (i.e., the different illuminances would have differential impact on cones but a similar, saturating impact on rods). During the experiment, each of the two lamps produced each of the three illuminances once (six different light exposures), with the order of the exposures being randomized separately for each participant (randomization from Random.org), with an additional 1-min light exposure from the standard LED lamp being the first in all cases (calibration). Pupil dynamics were analyzed offline with ViewPoint EyeTracker (Arrington Research, Scottsdale AZ) and macros developed in Excel (v.16.0.4549.1000, Microsoft, Redmond WA). Following the final 10-min dark exposure to assess pupil redilation, the experiment was concluded, and the participant was discharged from the study.
For each participant, the eye with the best pupillometric signal-to-noise ratio was selected. Artifacts (notably, eye blinks) were manually removed and pupil data were smoothed (Loess with fourth-degree polynomial) before analyses. Pupil size was calculated as the width of an elliptical contour fitted by the Arrington software. Baseline pupil size was calculated as the median pupil size during the $25 \mathrm{~s}$ of darkness preceding each light exposure. Pupil constriction was baseline-adjusted such that: $\%$ pupil constriction from baseline $=\left(\frac{\text { baseline pupil size-pupil size }}{\text { baseline pupil size }}\right) \times 100$ (Joyce et al., 2016). Multiple pupillometric parameters were calculated, including: peak phasic constriction (largest acute reduction in pupil size), sustained pupil constriction (median of constricted pupil size 10 post light-onset to $5 \mathrm{~s}$ pre light-offset), and the post-illuminance pupil response (PIPR), which has been shown to be representative of ipRGC activity (Adhikari et al., 2015) (Fig. 2) and calculated as the percent pupil constriction from baseline $6 \mathrm{~s}$ after light-offset. The time from light onset to peak constriction (constriction speed) and from light offset to $90 \%$ of baseline (re-dilation speed) were also calculated. All pupil analyses were conducted blind to the specific illuminance and lamp being tested.

\section{Study 2}

We examined a separate 10 participants (five male, five female) in a randomized, double-blind cross-over trial. Six were Caucasian and four were Asian. They were aged $25-35$ years $(29 \pm 3.0$ years, mean \pm SD). All participants were in good health and passed the same screening as reported in Study 1.

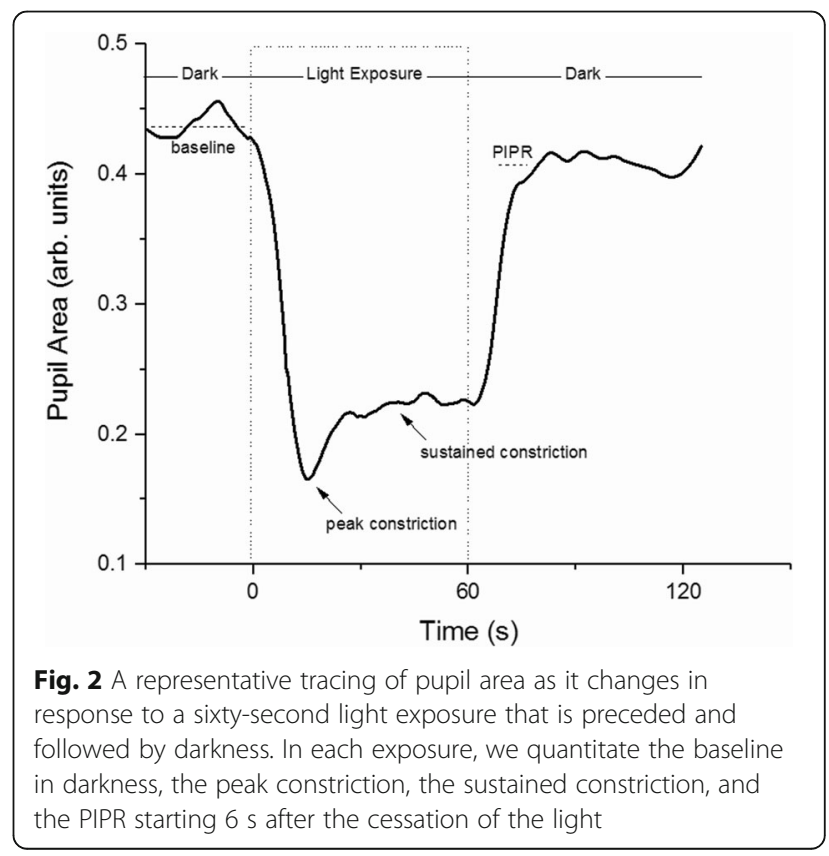


Following consent and screening, participants were scheduled for two overnight stays at the Zeitzer laboratory at the VA Palo Alto Health Care System. Each stay was at least one week apart. During the week prior to entry into the laboratory, participants kept a regular sleep/wake schedule such that all bed and wake times were within $\pm 30 \mathrm{~min}$ of a participant-set target time and 7-9 h apart. Compliance with this schedule was confirmed through examination of self-reported sleep logs and continuous wrist actigraphy (Motionlogger, Ambulatory Monitoring, Ardsley NY), a useful proxy for determining sleep/wake patterns (Ancoli-Israel et al., 2003). One divergence from the schedule was permitted - otherwise participants were rescheduled. Habitual bedtime was calculated as the midpoint of the at-home sleep schedule minus four hours. The timing of all laboratory procedures were based off this calculation. For young individuals with a regular sleep/wake schedule and an intermediate chronotype, determination of habitual sleep timing is a useful approximation of the position of the endogenous circadian clock (Duffy et al., 1998).

Participants arrived at the laboratory for the overnight study approximately four hours prior to their target bedtime. Once an accurate sleep schedule was verified, participants were brought to the room in which they would spend the next $14 \mathrm{~h}$. The room is specially designed for the conduct of circadian studies. There are no windows and all lighting is controlled by a panel outside of the room. The walls are painted with a highly reflective titanium-dioxide-based white paint and all of the surfaces are white or covered in white sheets. Upon entry into the room, the lights were dimmed $(<10 \mathrm{~lx}$ in any angle of gaze, ILT1700 Research Photometer, International Light Technologies, Peabody MA). Room lighting was produced with evenly spaced fluorescent lamps (Philips F32 T8, $3500 \mathrm{~K}$ ) controlled by an electronic ballast. For the next $14 \mathrm{~h}$, participants lay in bed and rested in either a semirecumbent (during periods of wake) or flat (during periods of sleep) position. Saliva samples were collected every $30 \mathrm{~min}$ (Salivette, Sarstedt, Newton NC), with $90 \mathrm{~mL}$ of water being provided after each saliva sample and removed $10 \mathrm{~min}$ prior to the subsequent saliva collection. Collection of saliva under conditions of constant dim light and constant posture is both sufficient and necessary for the accurate collection of unattenuated concentrations of melatonin (Duffy \& Dijk, 2002). A Stanford Sleepiness Scale (SSS) (Hoddes et al., 1973) was obtained hourly following a saliva sample collection. The SSS is a Likert-like scale from 1 to 7 , with higher numbers indicating greater sleepiness.

At habitual bedtime, the overhead dim light was turned off and the experimental light was turned on for three hours. The experimental light was fixed to the foot of the bed in an aluminum reflector such that the front of the light was facing the participant. During the experimental light exposure, saliva sample collection frequency was increased to every $20 \mathrm{~min}$. The experimental light was one of two conditions: a standard white light LED or a bluedepleted white LED, both identical to those used in Study 1 (Fig. 1). Both lights were calibrated to a target of $150 \mathrm{~lx}$ at corneal level in a typical angle of gaze. By matching the lux units, the lights should have had a similar impact on image forming perception. The visit number ( 1 or 2 ) during which participants received the standard or blue-depleted white light was determined a priori through a random number generator (Random.org). The allocation was double-blind as neither the participant nor the laboratory technician administering the light knew which bulb was the standard and which was the bluedepleted. There was no obvious difference in the perceptual quality or color of the light emitted from the two lamps in the laboratory environment and the lamps were designated as " $\mathrm{A}$ " and " $\mathrm{B}$ " by the primary investigator (JMZ) - the laboratory technician was unaware of the matching of the $\mathrm{A} / \mathrm{B}$ designation and the type of lamp being used. During the three hours of experimental light exposure, participants were kept awake by a laboratory technician and asked to look at the LED lamp, alternating every ten minutes between a gaze fixed at the lamp and free gaze around the room. During both the free and fixed gaze components, participants were not permitted to avoid light exposure by hiding their eyes or directing their gaze downward (e.g., no reading was allowed). Illuminance during the fixed and free periods was recorded with the ILT1700 Research Photometer at the end of each of these periods, estimating the average angle of gaze. Three hours after habitual bedtime, all lighting was turned off and the participant was allowed to sleep ad libitum. Upon arising, the participant was given a standard hospital breakfast, and could leave the unit.

Saliva samples were immediately frozen $\left(-20{ }^{\circ} \mathrm{C}\right)$ and placed in storage at $-80{ }^{\circ} \mathrm{C}$ within one week of collection. Saliva samples were assayed as a single batch in duplicate using a salivary melatonin enzyme-linked immunosorbent assay (ALPCO, Salem NH) per the manufacturer's instructions. Published intra-and inter-assay coefficients of variation are $6.1-13.0 \%$ with an assay sensitivity of $0.3 \mathrm{pg} / \mathrm{mL}$. The assay microplate was read using a Multiskan FC Microplate Photometer (Thermo Scientific, Waltham MA). One of the 10 participants had a failed melatonin assay and insufficient saliva to conduct a repeat assay; the melatonin data from this participant were excluded. The three hours prior to habitual sleep onset, during which participants were in dim light, was denoted as the "baseline". Melatonin levels were expected to be rising to their elevated nocturnal levels during the baseline. The three hours after habitual bedtime, during which participants were exposed to the experimental light, was 
denoted as the "light exposure". Melatonin data during the baseline and light exposure were integrated over time using the trapezoidal method. Integrated melatonin concentrations during each hour of the light exposure was separately calculated.

Statistical analyses, as specified below, were performed using either OriginPro 2017 (v.b9.4.0220, OriginLab Corporation, Northampton MA) or Excel (v.16.0.4549.1000, Microsoft, Redmond WA). Z-score transformation was done for visualization purposes only (see Fig. 3). The mean and standard deviation of each participant's melatonin data were determined and the individual values were $\mathrm{z}$ score transformed as $\mathrm{z}=(\mathrm{X}-\mu) / \sigma$, such that $\mathrm{X}=$ sample value, $\mu=$ population mean, and $\sigma=$ population standard deviation.

\section{Results}

\section{Lamp characteristics}

The standard white LED has broad coverage of the visible wavelengths, with a major peak at $607 \mathrm{~nm}$ (orange) and a minor peak at $461 \mathrm{~nm}$ (blue) (Fig. 1). The bluedepleted white LED has similar coverage, but lacks blue wavelengths and has a major peak at $413 \mathrm{~nm}$ (violet) and secondary peaks at $641 \mathrm{~nm}$ (red) and $551 \mathrm{~nm}$ (green) (Fig. 1). Due to the difference in the lamp output in the blue region of visible light (450-495 nm), at the target of $150 \mathrm{~lx}$, the corresponding $\alpha$-opic melanopic lux is 77.0 for the standard white LED and 46.0 for the bluedepleted white LED (Lucas et al., 2014), a 40\% reduction in the drive on melanopsin.

\section{Study 1}

Following the stable pupil size observed under conditions of darkness, the pupil rapidly constricted to a minimum size in response to both lights (Fig. 2). There was a slight but stable relaxation of the constriction over the next $55 \mathrm{~s}$, remaining throughout the remainder of the light stimulus. At the cessation of the light stimulus, the pupil size redilated to approximately $90 \%$ of baseline size within approximately $9 \mathrm{~s}$. The pupil remained constricted greater than baseline in darkness for several seconds before returning to full dilation. Baseline (darkness) pupil size was not different within participants $(p=0.15$, repeated measure one-way ANOVA). Visual and statistical inspection of the plots revealed no differences in pupillometric parameters between the higher $(100,140 \mathrm{~lx})$ lighting conditions of the two lamps (Table 1). Responses to $32 \mathrm{~lx}$, however, appeared to be lamp-specific. There was no difference between the lamps in terms of phasic $(p=0.51$, paired t-test) or sustained ( $p=0.42$, paired t-test) constriction, nor was there a difference in the constriction speed ( $p=0.36$, paired $\mathrm{t}$-test). The re-dilation speed in response to the blue-depleted white light at $32 \mathrm{~lx}$ was, however, $58 \%$ faster $(p<0.05$, paired t-test) and the PIPR was also reduced by $45 \%$ with the blue-depleted white light $(p<0.05$, paired t-test) as compared to the standard white LED (Table 1).

\section{Study 2}

The same lamps were used in Study 2 as were used in Study 1 (Fig. 1), but were calibrated prior to the experiment to produce $150 \mathrm{~lx}$ at corneal level. During the study, exposure to the standard white LED was $150 \pm$ $3.24 \mathrm{~lx}$ during the fixed gaze and $151 \pm 2.70$ during the free gaze. Exposure to the blue-depleted white LED was $153 \pm 4.60 \mathrm{~lx}$ during the fixed gaze and $152 \pm 5.05$ during the free gaze. There was no difference in the photopic illuminance received during the fixed and free $(p=0.11$, repeated measure two-way ANOVA) or between the blue-depleted and standard white LED lamps $(p=0.15$, repeated measure two-way ANOVA).

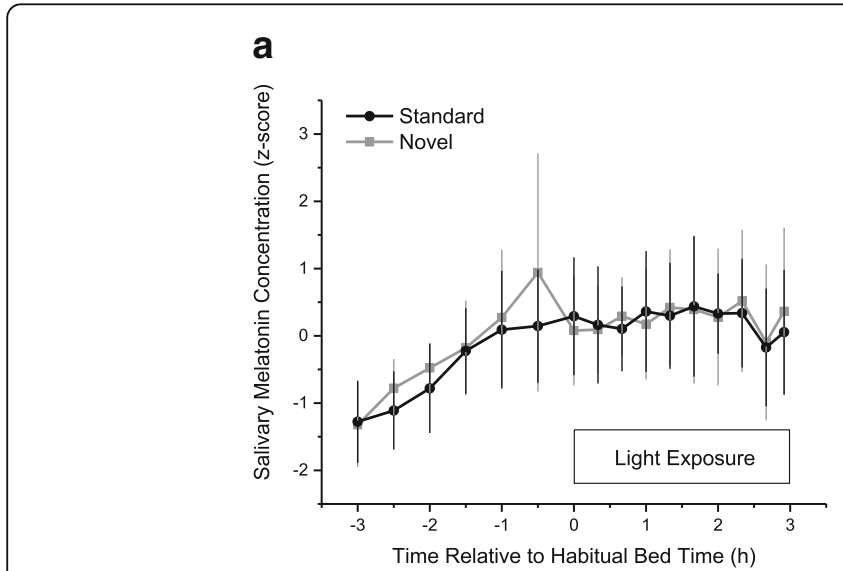

b

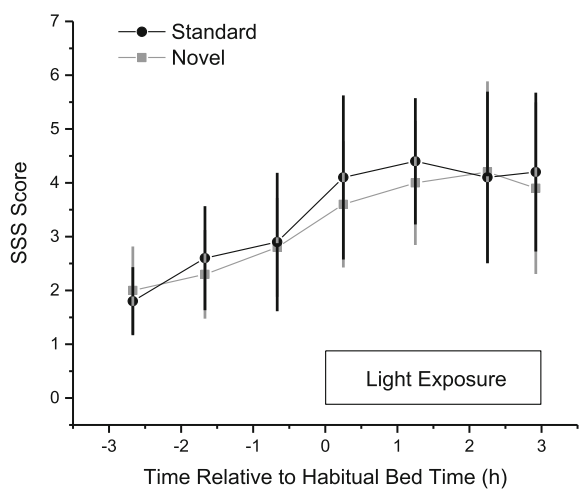

Fig. 3 Changes in salivary melatonin concentration (a) and SSS values (b) during the baseline $(<10 \mid x,-3 \rightarrow 0 h)$ and experimental light exposure $(150 \mathrm{~lx}, 0 \rightarrow 3 \mathrm{~h}$ ) for both the blue-depleted (grey) and standard (black) white LED lamps. Melatonin data were $\mathrm{z}$-score transformed and averaged prior to plotting. Mean \pm SD are shown 
Table 1 Pupil responses to light

\begin{tabular}{|c|c|c|c|c|c|c|}
\hline \multicolumn{7}{|c|}{ Blue-Depleted White LED } \\
\hline Illuminance & Baseline (arb. unit) & Phasic max constriction (\%) & Sustained constriction (\%) & Constriction Slope (\%/s) & Dilation Slope (\%/s) & PIPR 6 s (\%) \\
\hline $32 \mathrm{~lx}$ & $0.46 \pm 0.072$ & $-64 \pm 5.8$ & $-56 \pm 4.2$ & $-8.2 \pm 2.3$ & $7.7 \pm 1.0^{*}$ & $-11 \pm 6.7^{*}$ \\
\hline $100 \mathrm{~lx}$ & $0.43 \pm 0.095$ & $-63 \pm 8.3$ & $-54 \pm 6.8$ & $-8.5 \pm 3.5$ & $7.1 \pm 4.1$ & $-10 \pm 13$ \\
\hline $140 \mathrm{~lx}$ & $0.48 \pm 0.056$ & $-67 \pm 5.8$ & $-60 \pm 4.7$ & $-8.5 \pm 2.1$ & $6.4 \pm 3.2$ & $-19 \pm 18$ \\
\hline \multicolumn{7}{|c|}{ Standard White LED } \\
\hline Illuminance & Baseline (arb. unit) & Phasic max constriction (\%) & Sustained constriction (\%) & Constriction Slope (\%/s) & Dilation Slope (\%/s) & PIPR 6 s (\%) \\
\hline $32 \mathrm{~lx}$ & $0.45 \pm 0.087$ & $-62 \pm 10$ & $-53 \pm 11$ & $-7.2 \pm 2.5$ & $4.9 \pm 1.8$ & $-20 \pm 3.5$ \\
\hline $100 \mathrm{~lx}$ & $0.45 \pm 0.097$ & $-65 \pm 4.3$ & $-57 \pm 3.3$ & $-9.2 \pm 2.1$ & $7.8 \pm 2.8$ & $-10 \pm 11$ \\
\hline $140 \mathrm{~lx}$ & $0.45 \pm 0.094$ & $-66 \pm 4.5$ & $-57 \pm 6.5$ & $-7.8 \pm 2.3$ & $5.1 \pm 2.7$ & $-23 \pm 16$ \\
\hline
\end{tabular}

Comparison of pupil responses to two different lamps - a blue-depleted white LED (top) and a standard white LED (bottom) - to three different illuminances of light. Light-induced changes in pupil size were normalized to percent of baseline pupil size. ${ }^{*} p<0.05$ using a paired Student t-test. Data are presented as mean $\pm S D$

Melatonin concentrations during the baseline portion prior to exposure to the blue-depleted or standard white LED lighting were similar ( $p=0.33$, paired t-test) as the expected rise of melatonin occurred in the hours prior to habitual bed time (Fig. 3a). There was no obvious impact of either light source on salivary melatonin concentrations (Fig. 3a). Melatonin concentrations during the full exposure $(p=0.40$, paired t-test) or during any single hour of exposure (p's $>0.37$, paired t-tests) to the blue-depleted white light was indistinguishable from that observed during the same time of exposure to the standard white light.

SSS scores during the baseline portion prior to exposure to the blue-depleted or standard white LED lighting were similar ( $p=0.78$, paired $\mathrm{t}$-test). The expected rise in subjective sleepiness occurred around the time of habitual bedtime and this level of sleepiness was maintained throughout the three hours of light exposure (Fig. 3b). There was no obvious difference in the impact of the two light sources on SSS scores ( $p=0.21$, paired t-test).

\section{Discussion}

At a low illuminance of $32 \mathrm{~lx}$, the blue-depleted white LED light ( $40 \%$ lower melanopsin stimulation) had significantly less of an impact on the post-illumination pupil response (PIPR) than a broad spectrum white LED light, and did so without impacting melatonin or subjective sleepiness. At higher illuminances $(\geq 100 \mathrm{~lx})$, there were no differences in light-induced PIPR, melatonin suppression, or relief of subjective sleepiness between the two light sources.

Our pupil findings are consistent with the theory that the PIPR is driven by melanopsin. At the lower illuminance tested, PIPR was reduced in response to the light that had $40 \%$ less drive on melanopsin. We did not, however, observe an impact of the lights on phasic constriction, which is also thought to be influenced by melanopsin. The partial (40\%) reduction in melanopic drive and the relatively small number of participants may have contributed to our inability to detect such a difference. We also used an extended (60 s) light stimulation; shorter light stimulations (e.g., $1 \mathrm{~s}$ ) may have revealed more differences in PIPR at the higher intensities. The pupil responses to light that are dependent upon cones were not differentially impacted by the two lights tested.

None of the other non-image forming functions that we tested, however, were impacted by the reduction of melanopsin drive in the blue-depleted white light. Previous studies have indicated that $150 \mathrm{~lx}$ of white light is sufficient to suppress melatonin and decrease subjective sleepiness (Zeitzer et al., 2000; Cajochen et al., 2000). It must be noted, however, that these measures of lightinduced changes were conducted after extended $(>40 \mathrm{~h}$ ) exposure to no greater than dim light. In the current study, participants arrived to the lab after exposure to real-world environments and were in $\operatorname{dim} \operatorname{light}(<10 \mathrm{~lx})$ for only three hours prior to the experimental light exposure. Previous studies (Smith et al., 2004; Chang et al., 2011; Rufiange et al., 2007; Zeitzer et al., 2011) have demonstrated that the impact of light on nonimage forming functions, such as those presented in this report, is sensitized by previous exposure to dim light. It is, therefore, possible that at increased intensities we may have observed the expected changes in melatonin and alertness. Under the ecologically relevant conditions to which most individuals are exposed, however, we do not observe meaningful changes in melatonin or alertness after exposure to normal room light intensities. Individuals who lack exposure to bright indoor or outdoor light, however, might benefit from exposure to blue-depleted white light (Rahman et al., 2017).

Given the previous literature, had we sensitized the non-image forming system with many hours of dim or room light prior to exposure or increased the intensity of the experimental light exposure, we might have observed a difference in the impact of the two lamps on 
melatonin suppression and subjective alertness. We did not design the experiment as such since previous studies had already established the biological capacity of the nonimage forming photoreceptive system to have a peak response to long duration blue light. We, rather, were concerned with the potential real-world use of white light lamps that had diminished impact on melanopsin and, at least for subjective sleepiness and melatonin suppression, we did not observe an advantage of the blue-depleted white LED lamp. We did not explicitly examine objective alertness or circadian phase shifting here, but these two processes operate in a similar intensity range as subjective alertness and melatonin suppression (Zeitzer et al., 2000; Cajochen et al., 2000) and might have similar outcomes. Future research could examine the utility of such lamps as sleep-permissive light sources in environments in which individuals have consistent exposure to lower levels of daytime lighting (e.g., submarines, winter time in extreme northern latitudes, all day low intensity office lighting).

\section{Conclusions}

After exposure to a real-world daytime lighting environment, except for pupil responses to moderately dim light, there are no difference in non-imaging forming responses to broad spectrum white light and a broad spectrum white light with reduced blue content.

\section{Abbreviations}

ANOVA: Analysis of variance; ipRGC: Intrinsically photosensitive retinal ganglion cells; LED: Light emitting diode; PIPR: Post-illumination pupillary response; SSS: Stanford Sleepiness Scale

\section{Acknowledgements}

We wish to thank Ms. Chun-Ping Liao for conduct of the melatonin assays and Dr. Aurélien David of Soraa for helpful discussions of lighting and facilitating the implementation of this study.

\section{Funding}

This research was conducted using a grant from Soraa Inc. The company provided both material and financial support. The company was not involved in the design, conduct, or analysis of the research, nor were they involved in the writing of this manuscript.

\section{Availability of data and materials}

All data generated or analyzed during this study are included in this published article.

\section{Authors' contributions}

JMZ designed and analyzed the study. RPN analyzed the pupil data. C-A Wang conducted the study. MK assisted in data analyses. All authors read and approved the final manuscript.

\section{Ethics approval and consent to participate}

All procedures were pre-approved by the Stanford University Institutional Review Board (IRB \#36326). All participants signed a consent form prior to the initiation of any screening or study procedures.

\section{Consent for publication}

Not applicable

\section{Competing interests}

The authors declare no competing interests directly relevant to this manuscript. None of the authors have any financial holdings or relationships with the funder, Soraa (see below).

\section{Publisher's Note}

Springer Nature remains neutral with regard to jurisdictional claims in published maps and institutional affiliations.

\section{Author details \\ 'Department of Psychiatry and Behavioral Rhythms, Stanford University, Stanford, CA 94305, USA. ${ }^{2}$ Mental Illness Research Education and Clinical Center, VA Palo Alto Health Care System, Palo Alto, CA 94304, USA. ${ }^{3}$ Department of Visual Neurosciences, Singapore Eye Research Institute, Singapore 169856, Singapore. ${ }^{4}$ Present address: Connecticut College, New London, CT, USA.}

Received: 5 January 2018 Accepted: 11 February 2018

Published online: 05 March 2018

\section{References}

Adhikari P, Zele AJ, Feigl B. The post-illumination pupil response (PIPR). Invest Ophthalmol Vis Sci. 2015;56:3838-49.

Alpern M, Campbell FW. The spectral sensitivity of the consensual light reflex J Physiol. 1962;164:478-507.

Ancoli-Israel S, Cole R, Alessi C, Chambers M, Moorcroft W, Pollack CP. The role of actigraphy in the study of sleep and circadian rhythms. Sleep. 2003:26:342-92.

Berson DM, Dunn FA, Takao M. Phototransduction by retinal ganglion cells that set the circadian clock. Science. 2002;295:1070-3.

Brainard GC, Hanifin JP, Greeson JM, Byrne B, Glickman G, Gerner E, Rollag MD. Action spectrum for melatonin regulation in humans: evidence for a novel circadian photoreceptor. J Neurosci. 2001;21:6405-12.

Buysse DJ, Reynolds CF III, Monk TH, Berman SR, Kupfer DJ. The Pittsburgh sleep quality index: a new instrument for psychiatric practice and research. Psychiatry Res. 1989;28:193-213.

Cajochen C, Zeitzer JM, Czeisler CA, Dijk D-J. Dose-response relationship for light intensity and ocular and electroencephalographic correlates of alertness in humans. Beh Brain Res. 2000;115:75-83.

Chang AM, Scheer FA, Czeisler CA. The human circadian system adapts to prior photic history. J Physiol. 2011;589:1095-102.

Czeisler CA, Kronauer RE, Allan JS, Duffy JF, Jewett ME, Brown EN, Ronda JM. Bright light induction of strong (type 0) resetting of the human circadian pacemaker. Science. 1989:244:1328-33.

Duffy JF, Dijk D-J. Getting through to circadian oscillators: why use constant routines? J Biol Rhythm. 2002:17:4-13.

Duffy JF, Dijk D-J, Klerman EB, Czeisler CA. Later endogenous circadian temperature nadir relative to an earlier wake time in older people. Am J Phys. 1998;275:R1478-87.

Gooley JJ, Rajaratnam SM, Brainard GC, Kronauer RE, Czeisler CA, Lockley SW. Spectral responses of the human circadian system depend on the irradiance and duration of exposure to light. Sci Transl Med. 2010;2:31ra33.

Hoddes E, Zarcone V, Smythe H, Phillips R, Dement WC. Quantification of sleepiness: a new approach. Psychophysiology. 1973;10:431-6.

Horne JA, Östberg O. A self-assessment questionnaire to determine morningness-eveningness in human circadian rhythms. Intl J Chronobiol. 1976:4:97-110.

Ishihara S. Ishihara's tests for colour deficiency. Tokyo: Kanehara Trading Inc.; 2007.

Joyce DS, Feigl B, Zele AJ. The effects of short-term light adaptation on the human post-illumination pupil response. Invest Ophthalmol Vis Sci. 2016;57: $5672-80$

Lucas RJ, Peirson SN, Berson DM, Brown TM, Cooper HM, Czeisler CA, Figueiro MG, Gamlin PD, Lockley SW, O'Hagan JB, et al. Measuring and using light in the melanopsin age. Trends Neurosci. 2014;37:1-9.

McBean AL, Najjar RP, Schuchard RA, Hall CD, Wang C-A, Ku B, Zeitzer JM. Standing balance and spatiotemporal aspects of gait are impaired upon nocturnal awakening in healthy late middle-aged and older adults. J Clin Sleep Med. 2016;12:1477-86. 
Nelson DE, Takahashi JS. Sensitivity and integration in a visual pathway for circadian entrainment in the hamster (Mesocricetus auratus). J Physiol. 1991; 439:115-45.

Newman LA, Walker MT, Brown RL, Cronin TW, Robinson PR. Melanopsin forms a functional short-wavelength photopigment. Biochemist. 2003;42:12734-8.

Radloff LS. The CES-D scale: a self-report depression scale for research in the general population. Appl Psychol Meas. 1977;1:385-401.

Rahman SA, St Hilaire MA, Lockley SW. The effects of spectral tuning of evening ambient light on melatonin suppression, alertness and sleep. Physiol Behav. 2017;177:221-9.

Rufiange M, Beaulieu C, Lachapelle P, Dumont M. Circadian light sensitivity and rate of retinal dark adaptation in indoor and outdoor workers. J Biol Rhythm. 2007;22:454-7.

Santhi N, Thorne HC, van der Veen DR, Johnsen S, Mills SL, Hommes V, Schlangen LJM, Archer SN, Dijk D-J. The spectral composition of evening light and individual differences in the suppression of melatonin and delay of sleep in humans. J Pineal Res. 2011;53:47-59.

Smith KA, Schoen MW, Czeisler CA. Adaptation of human pineal melatonin suppression by recent photic history. J Clin Endo Metab. 2004:89:3610-4.

Zeitzer JM, Dijk D-J, Kronauer RE, Brown EN, Czeisler CA. Sensitivity of the human circadian pacemaker to nocturnal light: melatonin phase resetting and suppression. J Physiol. 2000;526:695-702.

Zeitzer JM, Friedman L, Yesavage JA. Effectiveness of evening phototherapy for insomnia is reduced by bright daytime light exposure. Sleep Med. 2011;12:805-7.

\section{Submit your next manuscript to BioMed Central and we will help you at every step:}

- We accept pre-submission inquiries

- Our selector tool helps you to find the most relevant journal

- We provide round the clock customer support

- Convenient online submission

- Thorough peer review

- Inclusion in PubMed and all major indexing services

- Maximum visibility for your research

Submit your manuscript at www.biomedcentral.com/submit

C) Biomed Central 